\title{
An agent-based simulation framework to evaluate urban logistics schemes
}

\author{
Wouter van Heeswijk, Martijn Mes and Marco Schutten \\ University of Twente \\ Department of Industrial Engineering and Business Information Systems \\ P.O. Box 217, 7500 AE Enschede, The Netherlands \\ $\{$ w.j.a.vanheeswijk,m.r.k.mes,m.schutten\}@utwente.nl
}

\begin{abstract}
Inefficient urban freight transport has a negative impact on both livability in cities and profit margins in the supply chain. Urban logistics schemes, consisting of governmental policies and company initiatives, attempt to address these problems. However, successful schemes are difficult to realize due to the divergent objectives of the agents involved in urban logistics. Traditional optimization techniques fall short when evaluating schemes, as they do not capture the required change in behavior of autonomous agents. To properly evaluate schemes, we develop an agent-based simulation framework that assesses the interaction between five types of autonomous agents. Compared to existing studies in this field, we contribute by (i) explicitly including company-driven initiatives, and (ii) adopting a supply chain-wide perspective. We illustrate the working of our framework by testing a number of schemes on a virtual network.
\end{abstract}

Keywords: urban logistics, agent-based simulation, logistics schemes

\section{Introduction}

The need to organize urban freight transport in an efficient manner is becoming increasingly important. Projections indicate a strong growth in the population of urban areas (both relatively and in an absolute sense) [21], resulting into a larger demand for goods. Other trends affecting urban freight transport are e-commerce, just-in-time approaches at retailers, higher dispersion of delivery locations, and increased service levels (e.g., shorter lead times, narrow delivery slots) $[2,5]$. As a result, shippers and carriers need to deal simultaneously with increasing shipment frequencies and decreasing order volumes, making it difficult for individual agents to transport goods efficiently [7]. As a result, trucks are often forced to carry low volumes and make inefficient delivery tours. This inefficiency contributes to external costs such as congestion, emissions, and noise hindrance, thereby negatively affecting the quality of life in urban areas. Furthermore, it reduces the profitability of the agents in the supply chain. In response to these developments, there is a strong interest in city logistics initiatives. Such initiatives, commonly called schemes, consist of one or more forms of companydriven change and governmental policies, with the aim to improve efficiency 
and/or reduce external costs [3]. Urban consolidation centers (UCCs) have a central role in most schemes, facilitating order bundling and efficient last-mile distribution. In Fig. 1, we give an example of the typical network setting that we consider. Since agents that handle large volumes often have access to the economies of scale and expertise to optimize their transport processes, our focus is on agents that handle small volumes, who are affected the most by the aforementioned trends.

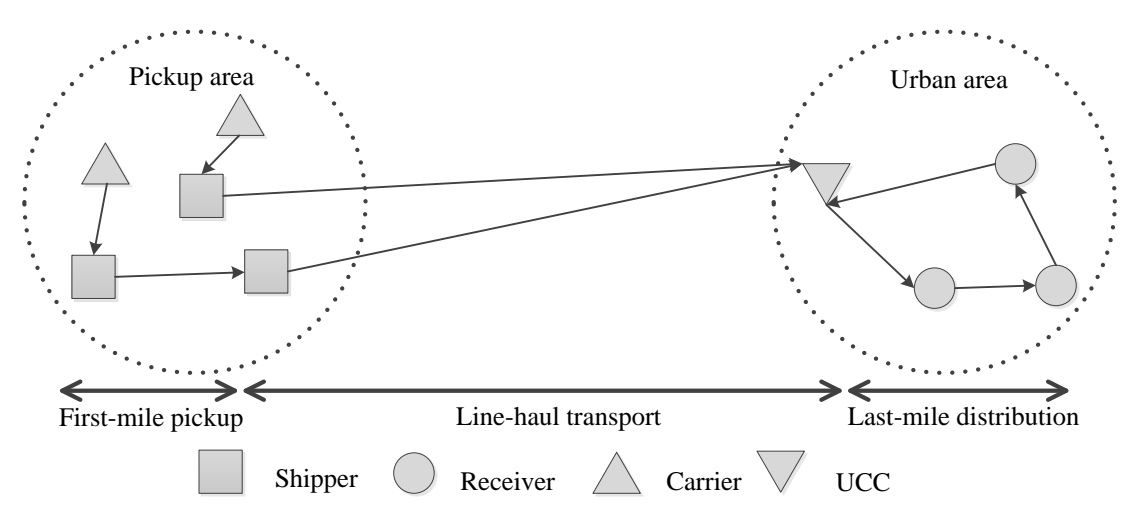

Fig. 1. Example of a network with a UCC facilitating last-mile distribution

Despite the eminent need for better organizing urban logistics, the vast majority of schemes fail after a short life-span [8]. A key reason for this is that the involved agents typically have divergent objectives, making it difficult to find solutions to which all agents are willing to commit [4]. Administrators often attempt to generate commitment by providing financial incentives in the form of subsidies [22]. However, such solutions are often not sustainable once the subsidies are halted. Another problem is that schemes are often implemented with little preliminary analysis, thereby not adequately evaluating their system-wide impact [16]. Finally, studies often focus solely on the processes within the city boundaries, while last-mile distribution accounts for a small part of the supply chain. As such, they ignore the impact of upstream decisions. Of particular importance is the allocation of slack in the chain; holding freight early in the supply chain may improve vehicle fill rates, but reduces flexibility for the UCC in last-mile distribution. In our framework, we explicitly address these aspects.

A key success factor for urban logistics schemes is the right combination of company involvement and governmental policies [8]. Agents must be willing to permanently change their behavior, without requiring an ongoing external cash flow. Traditional optimization techniques may be used to find viable system-wide solutions, yet these are not guaranteed to be stable when depending on multiple decision makers. Furthermore, it is difficult to evaluate the impact of combining multiple measures into a scheme. Agent-based simulation studies are suitable to 
evaluate such schemes, as they are capable of monitoring and altering the behavior of autonomous agents under conditions that can be flexibly adjusted [20]. With this study, we provide an agent-based simulation framework to evaluate the effectiveness of urban logistics schemes that include both governmental policies and company-driven initiatives.

\section{Literature Review}

For recent literature reviews on urban logistics, we refer to Anand et al. [2] and Bektaş et al. [4]. These reviews state that most studies focus on describing and evaluating existing initiatives, rather than optimizing. Only few papers adopt an operations research perspective [10]; these papers generally address (i) the positioning of UCCs and (ii) solution methods to one-echelon or two-echelon routing problems. Most urban logistics initiatives are characterized by the use of UCCs. Inbound trucks no longer need to enter the city center, but instead unload at a UCC, which is typically located at the edge of an urban area. Subsequently, goods can be bundled at the UCC, such that efficient tours can be made for the last-mile distribution. Furthermore, environment-friendly vehicles can be dispatched for last-mile distribution. Particularly for independent low-volume, high-frequency deliveries, UCCs could substantially improve performance.

Browne et al. [8] provide an elaborate overview of real-life UCC projects, and report that only few initiatives were able to remain in operation for multiple years. A key success factor is the involvement of commercial parties that share a common objective. UCCs yield the best results when involving a sufficiently large number of small, independent shippers and retailers, where lowvolume, high-frequency shipments are the norm. Government administrators are typically required to cover the capital expenses of the UCC. Furthermore, gains from policies could (partially) cover operational expenses. However, UCCs that heavily rely on subsidies are unlikely to succeed in the long run, as profit margins in logistics are too small to absorb a subsidy cut. Generally accepted financial models do not exist for UCCs [1]; it is often not clear how the costs of the UCC should be distributed among the administrator, receivers and carriers.

Quak [16] distinguishes four classes of initiatives in urban logistics. First, he considers improvements within the context of existing operations, in which he distinguishes between (i) governmental policies and (ii) company-driven initiatives. Second, he considers improvements that require changing the context of urban logistics, which he divides in (iii) physical infrastructure initiatives (including UCCs) and (iv) transport-reorganizing initiatives. Our present work is primarily focused on the first two classes, as they can be captured by the decisionmaking role of agents. Evaluating the latter two classes within our framework can be done by using various network configurations as input.

We discuss the aforementioned classes of initiatives, starting with governmental policies. To achieve norms on external costs, administrators encourage or enforce the desired behavior of agents in the supply chain by implementing policies. Common policies are vehicle access restrictions, time access restrictions, 
enforcing a minimum load factor, and road pricing [18]. Such policies typically favor small vehicles, e.g., heavy trucks face stricter time windows and higher costs than delivery vans. Individually, policies are often not enough to achieve the intended change. Agents should have viable alternatives to change their behavior, otherwise policies may even have results that oppose the intended effect [18]. For example, restrictive access times may increase the number of transport movements, forcing carriers to deploy additional vehicles.

Next, we describe the concept of company-driven change. Companies aim to increase transport efficiency mainly for economic reasons, but also because external costs become increasingly important for them. Forms of company-driven changes are, e.g., joint transportation by a coalition of carriers, deliveries outside normal delivery hours, or using the UCC as the delivery address [3]. The latter can be initiated by the receiver or by the carrier. For the carrier, cost savings (the costs of last-mile distribution are disproportionally high [11]) and legislative restrictions are the main reasons to use a UCC. For receivers, poor accessibility by truck and lower receiving costs (due to bundled deliveries) are reasons to consider delivery via the UCC [24].

Collaboration is notoriously difficult to realize in urban logistics. As the objectives of the agents in urban logistics are often divergent [4], system-wide optimization yields little practical insights. Techniques such as multi-criteria, multi-actor evaluation may yield more insights into the alignment of individual agents' objectives and feasible solutions [13]. However, such an evaluation does not guarantee commitment of the individual agents. Tanaguchi et al. [20] state that agent-based simulation is the most applicable method to study the behavior of and interaction between the various agents for urban logistics schemes. Agent-based simulation is not fit to study detailed interactions [4], yet is suitable to deduce generic insights on system performance. We mention some notable agent-based simulation studies in the field of urban logistics. Tamagawa et al. [19] perform an agent-based simulation, in which they heuristically solve a VRP and iteratively update the actions of the agents. They test the effects of road pricing and truck bans. Van Duin et al. [22] focus on the financial model and environmental impact of UCCs, taking into account UCC service fees, road pricing, and subsidies. Wangapisit et al. [25] evaluate the use of consolidation centers by imposing parking constraints and providing subsidies to carriers.

The contribution of our evaluation framework is twofold. First, we take into account the transport process outside the city. As last-mile distribution accounts for only part of the transport process, a narrow perspective does not properly assess the decisions made by shippers and carriers. Second, we explicitly include various forms of cooperation between companies, while existing studies tend to have a strong focus on testing governmental policies. As practice shows that successful schemes require both policies and commitment from companies, a framework including both aspects is essential for proper evaluation of these schemes. 


\section{Framework Design}

In this section, we outline the design of our agent-based simulation framework. We start by describing the roles of the agent types in Section 3.1. In Section 3.2, we formally define the state of the system, based on which we define the objective functions for each agent type and the corresponding performance indicators in Section 3.3. In Section 3.4, we discuss various policies for operational decisionmaking (e.g, dispatching, routing) during the simulation. Finally, Section 3.5 assesses the use of scenario analysis to incorporate tactical and strategic decisions (e.g., coalition forming, governmental policies) into the framework.

\subsection{Agent Types}

We design our agent-based simulation framework such that it can simultaneously evaluate company-driven initiatives and governmental policies. We focus on supply chains for a single city; extension of the framework to multiple cities is relatively straightforward. In such a context, harmonization of local policies and consolidation on the line haul are noteworthy challenges [16]. In our simulation, decisions are made at discrete moments in time. Five types of agents are distinguished: receivers, shippers, carriers, the UCC operator, and the administrator. We proceed to briefly describe their roles; Fig. 2 summarizes the array of actions, monetary flows and information flows between the agents. We note that the real-life counterparts of the agents are not necessarily rational decisionmakers, particularly when large changes in behavior are required. However, the simulation results yield insights into the behavioral effects of real-life agents, as such providing directions for change.

The receivers have a demand that is subject to some (stochastic) process, and they may order from multiple suppliers at a single decision moment. They order at fixed decision moments (e.g., twice per week); we assume that their ordering pattern already takes into account factors such as internal consolidation, storage costs, and stockouts. When ordering, receivers specify a delivery windows. Receivers can opt to select the UCC as their fixed delivery address. The shippers act on incoming orders, and hire carriers to transport orders. As carriers charge relatively less for higher volumes, shippers have an incentive to bundle multiple orders before shipping. However, the shippers should dispatch the orders in time, such that the carrier is able to meet the delivery windows. Line-haul carriers pick up goods at the shippers, and transport them either directly to the receivers or to the UCC. They may outsource the last-mile distribution to the UCC when this yields a financial benefit or is enforced by regulation. The carrier uses a price function based on volume and line-haul distance (i.e., the distance between cities, ignoring distance variations due to routing) that reflects economies of scale. In the typical setting we study, a dispatched truck will visit multiple cities during a single tour, yet we focus on a single city only. Consequently, the load destined for the city is generally much less than the truck's capacity. The UCC receives incoming goods, and is responsible for the last-mile distribution. At the UCC, orders from various carriers can be bundled, and may 
be temporarily held to account for future consolidation opportunities. Finally, the administrator can implement governmental policies to influence the behavior of agents. Since such policies are typically implemented for a longer time, we do this on a scenario basis. The financial gains stemming from these policies may be redistributed to subsidize agents; to keep the scheme sustainable we do not allow for external cash flows.

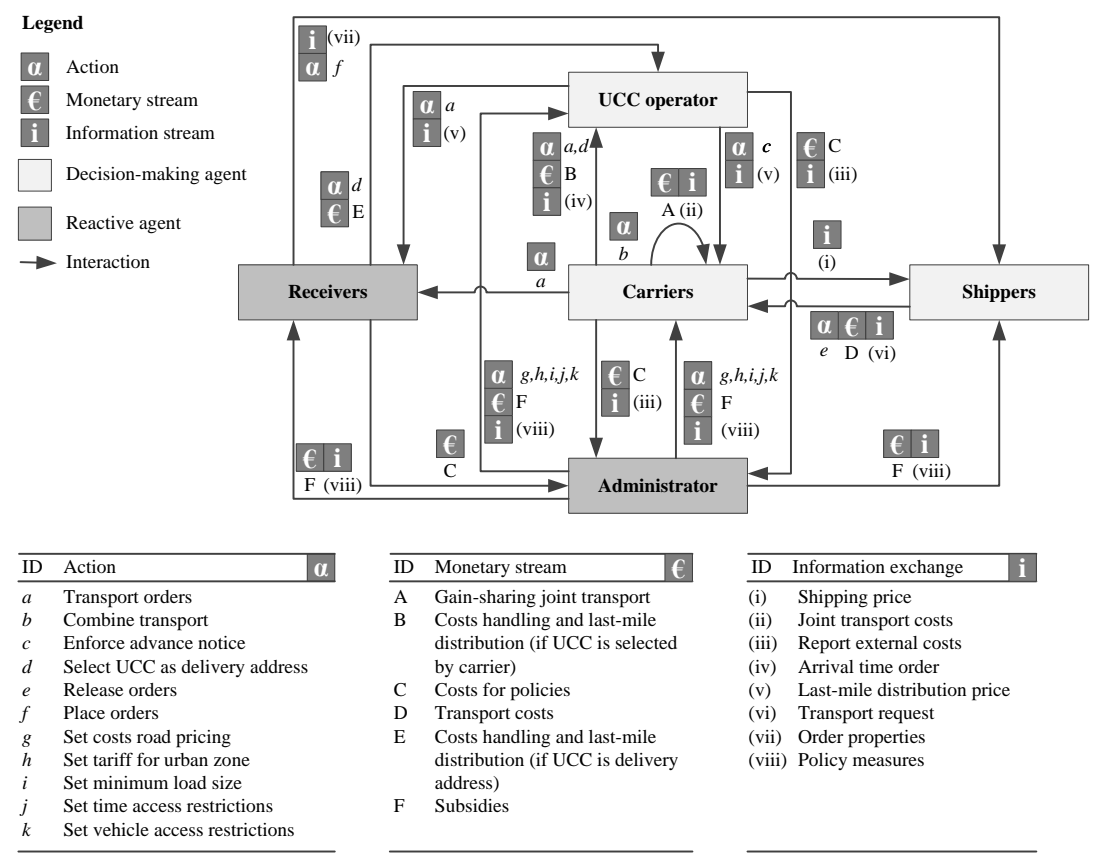

Fig. 2. Actions and interactions for all agent types

\subsection{System State}

In this section, we provide the notation required to define the state of the system and the actions of agents. Let $\mathcal{T}=\{0,1, \ldots, T\}$ be the set of discrete decision moments upon which agents can make decisions. Typically, the time between two decision moments will correspond to several hours, e.g., one decision moment in the morning, one in the afternoon, and one in the evening.

Let $\mathcal{C}$ be the set of carriers, $\mathcal{R}$ the set of receivers, and $\mathcal{S}$ the set of shippers. We assume a single UCC. The network is comprised by a vertex set $\mathcal{V}$ and a set 
of $\operatorname{arcs} \mathcal{A}$. Vertex set $\mathcal{V}$ is defined as $\mathcal{V}=\mathcal{V}^{R} \cup \mathcal{V}^{S} \cup \mathcal{V}^{C} \cup \mathcal{V}^{H}$, i.e., the set consists of subsets of locations of receivers, shippers, carriers and the UCC. As we have a single UCC, we have $\mathcal{V}^{H}=\left\{v^{u c c}\right\}$. A vertex in $\mathcal{V}^{C}$ indicates the starting point of a carrier, i.e., its home depot. Every arc $a \in \mathcal{A}$ connects a vertex pair $\left(v_{a}, v_{a}^{\prime}\right)$. Let $\mathcal{F}=\left\{\frac{1}{y}, \frac{2}{y}, \ldots, 1\right\}$ (with integer $y>1$ ) be the set of possible order volumes, expressed in terms of the capacity of the smallest vehicle (e.g., a delivery van). Based on the UCC dispatching problem defined by Van Heeswijk et al. [23], we define an order as a request to ship a certain load, with an order type being a unique combination of the delivery window $\left[t^{e}, t^{l}\right]$, the current position of the order $v \in \mathcal{V}$, (which indicates the agent responsible for handling the order at the current decision moment $t$ ), the receiver $r \in \mathcal{R}$, the carrier $c \in \mathcal{C} \cup \mathcal{H}$ (the UCC can be a carrier from the perspective of the receiver), the shipper $s \in \mathcal{S}$, the order volume $f \in \mathcal{F}$, and an indicator $\gamma \in\{0,1\}$ that specifies whether delivery takes place via the UCC ( $\gamma=1$ sets the UCC as the delivery address for the carrier). The indicator $\gamma$ can be specified either by the receiver (as a tactical decision) or by the carrier (as an operational decision); the agent specifying the index might incur a subsidy for this. We use $I_{t, t^{e}, t^{l}, v, r, c, s, f, \gamma}$ to denote the number of orders of a specific type. Not all indices are required by every agent for decision-making. For instance, the receiver will not specify which carrier delivers the order; this index is left blank until specified by the shipper. Furthermore, decisions may also transform order types, e.g., when the carrier decides to deliver via the UCC. The generic notation is applicable to all agents. We denote the number of orders per order type in the system at time $t$ as $I_{t}=\left[I_{t, t^{e}, t^{l}, v, r, c, s, f, \gamma}\right]_{\forall t^{e}, t^{l}, v, r, c, s, f, \gamma}$. In some form, every agent is faced with a dispatch decision based on $I_{t}$. Using generic notation, we denote the number of orders per order type dispatched at time $t$ as

$$
x_{t}=\left[x_{t, t^{e}, t^{l}, v, r, c, s, f, \gamma}\right]_{\forall t^{e}, t^{l}, v, r, c, s, f, \gamma},
$$

subject to

$$
\begin{aligned}
& x_{t, t^{e}, t^{l}, v, r, c, s, f, \gamma} \leq I_{t, t^{e}, t^{l}, v, r, c, s, f, \gamma} \quad \forall t^{e}, t^{l}, v, r, c, s, f, \gamma, \\
& x_{t, t^{e}, t^{l}, v, r, c, s, f, \gamma} \in \mathbb{N} \quad \forall t^{e}, t^{l}, v, r, c, s, f, \gamma .
\end{aligned}
$$

Both shippers and the UCC decide on the set of orders to dispatch at decision moment $t$, we denote these actions as $x_{t, v^{s}, s}^{s h p}$ and $x_{t, v u c c}^{u c c}$ respectively. The order of a receiver (based on the demand at time $t$ ) is described by $x_{t, v^{r}, r}$, while the shipment of the carrier is given by $x_{t, v^{c}, c}$.

We proceed with the notation required to denote routes. Let $\mathcal{Q}_{c}$ denote the set of vehicles operated by carrier $c$. A vehicle $q \in \mathcal{Q}_{c}$ has a vehicle capacity $\psi_{c, q} \in \mathbb{R}_{\geq 1}$, a line-haul travel speed $\tau_{c, q}^{l h} \in \mathbb{R}_{>0}$, and a last-mile travel speed $\tau_{c, q}^{l m} \in \mathbb{R}_{>0}$. To ease the notation, we assume that all routes starting at $t$ are completed at $t+1$. For the same reason, we do not explicitly include the pickup tour. We denote a route started by vehicle $q$ of carrier $c$ at time $t$ as $\delta_{t, c, q}^{c a r}=$ $\left\{\delta_{t, c, q}^{c a r, l h}, \delta_{t, c, q}^{c a r, l m}\right\}$, with the components referring to line-haul transport $(l h)$ and last-mile distribution $(l m)$ respectively. This distinction is used to assign distinct properties (e.g., fuel usage, road pricing, driver wage) to the associated travel 
distances $d^{l h}$ and $d^{l m}$. We let $\Delta_{t, c}^{c a r}$ denote the set of routes for carrier $c$ at decision moment $t$, and use $\Delta_{t}^{u c c}$ to describe the set of routes for the UCC. The UCC only has to deal with last-mile distribution, such that $\delta_{t, q}^{u c c}=\left\{\delta_{t, q}^{u c c, l m}\right\}$. We use $\Delta_{t}=\left[\Delta_{t, c}^{c a r}, \Delta_{t}^{u c c}\right]_{\forall c}$ to denote all routes starting at time $t$.

The system state at $t$ is given by $\left[I_{t}, \Delta_{t}\right]$; this description provides all information required for decision making and computing the performance indicators.

\subsection{Objective Functions and Key Performance Indicators}

In this section, we provide the objective functions and Key Performance Indicators (KPIs) of the agents. We start by introducing some notation required to describe the price- and cost functions. For a variety of parameters and variables, we use the superscript $h d$ to refer to costs for handling operations (e.g., (un)loading by the driver), $r c$ for receiving (e.g., lost work time, allocating goods), $s p$ for shipping (e.g., lost work time, loading operations), and $s b$ for income from subsidies. In our description, we restrict ourselves to subsidies for using the UCC. Price functions $P$ describe the income of agents and cost functions $C$ describe their expenses. The used order volumes depend on the context of the function. For example, the volume transported by carrier $c^{\prime}$ at time $t$ to the UCC is given by $f_{t, v^{c^{\prime}}, c^{\prime}, \gamma \mid \gamma=1}=\sum_{\forall t^{e}, t^{l}, r, s, f} x_{t, t^{e}, t^{l}, v^{c^{\prime}}, r, c^{\prime}, s, f, 1} \cdot f$. Other volumes are computed in a similar manner, using the corresponding subscripts.

For the carriers and UCCs, handling costs depend on the subsets of locations visited. A shipment may involve subsets of shippers $\mathcal{S}^{\prime} \subseteq \mathcal{S}$ and receivers $\mathcal{R}^{\prime} \subseteq$ $\mathcal{R} \cup \mathcal{H}$, this information is embedded in the route description. Note that the UCC is a receiver from the perspective of the carrier. The symbol $\alpha$-with the appropriate sub- and superscripts - refers to a fixed price- or cost component; similarly, the symbol $\beta$ refers to a variable price- or cost component. In Table 1, we provide price- and cost functions for corresponding to the agent's actions; for notational convenience we formulate all functions linearly. For the same purpose, we assume that carriers use homogenous fleets. Definitions are kept at a generic level; we define the objective functions of the actors and illustrating the main cost- and price components, without introducing excessive notational complexity.

Having defined the price- and cost functions, we now introduce the objective functions for the agents. The outcomes of these objective functions serve as KPIs for the agents. Although agents aim to optimize over the full planning horizon, they make periodic decisions based on incomplete information.

The objective of the shipper is to minimize the sum of transportation- and shipping costs. Shippers can influence these costs by selecting the set of orders to ship at every decision moment (denoted by $x_{t, v^{s}, s}^{s h p}$ ), and by selecting the cheapest carrier $c \in \mathcal{C}$ for the shipment.

$$
\min \sum_{t \in \mathcal{T}}\left(C_{t, s}^{s h p, t r}\left(f_{t, v^{s}, c, s}, d^{l h}\right)+C_{t, s}^{s h p, s p}\left(f_{t, v^{s}, c, s}\right)\right) .
$$

The objective of the UCC is to maximize profit, which is determined by the price charged by the UCC, subsidy income, receiving costs, and transport costs. 
Table 1. Price and cost functions for all decision-making agents

\begin{tabular}{|c|c|c|}
\hline \multicolumn{2}{|l|}{ Function } & Notes \\
\hline \multicolumn{3}{|l|}{ Receiver $(r e c)$} \\
\hline$P_{t, r}^{r e c, s b}\left(f_{t, v^{r}, r, \gamma \mid \gamma=1}\right)$ & $=\alpha_{r}^{r e c, s b}+\beta_{r}^{r e c, s b} \cdot f_{t, v^{r}, r, \gamma \mid \gamma=1}$ & $\begin{array}{l}\text { Income subsidies } \\
\text { (if } r \text { selects UCC) }\end{array}$ \\
\hline$C_{t, r}^{r e c, r c}\left(f_{t, v^{r}, r, c}\right)$ & $=\alpha_{r}^{r e c, r c}+\beta_{r}^{r e c, r c} \cdot f_{t, v^{r}, r, c}$ & Costs receiving shipment \\
\hline $\begin{array}{l}C_{t, r}^{r e c, l m}\left(f_{t, v^{r}, r, \gamma \mid \gamma=1}\right) \\
\text { Shipper }(s h p)\end{array}$ & $=P_{t}^{u c c, t r}\left(f_{t, v^{r}, r, \gamma \mid \gamma=1}\right)$ & Costs outsourcing UCC \\
\hline$C_{t, s}^{s h p, t r}\left(f_{t, v^{s}, c, s}, d^{l h}\right)$ & $=P_{t, c}^{t r}\left(f_{t, v^{s}, c, s}, d^{l h}\right)$ & Costs transportation \\
\hline $\begin{array}{l}C_{t, s}^{s h p, s p}\left(f_{t, v^{s}, c, s}\right) \\
\text { Carrier }(\text { car })\end{array}$ & $=\alpha_{s}^{s h p, s p}+\beta_{s}^{s h p, s p} \cdot f_{t, v^{s}, c, s}$ & Costs shipping \\
\hline$P_{t, c}^{c a r, s b}\left(f_{t, v^{c}, c, \gamma \mid \gamma=1}\right)$ & $=\alpha_{c}^{c a r, s b}+\beta_{c}^{c a r, s b} \cdot f_{t, v^{c}, c, \gamma \mid \gamma=1}$ & $\begin{array}{l}\text { Income subsidies } \\
\text { (if } c \text { selects UCC) }\end{array}$ \\
\hline$P_{t, c}^{c a r, t r}\left(f_{t, v^{c}, c}, d^{l h}\right)$ & $=\alpha_{c}^{c a r, t r}+\beta_{c}^{c a r, t r} \cdot f_{t, v^{c}, c} \cdot d^{l h}$ & Price shipping \\
\hline$C_{t, c}^{c a r, t r}\left(d^{l h}, d^{l m}, \mathcal{S}^{\prime}, \mathcal{R}^{\prime}\right)$ & $\begin{aligned}= & \alpha_{c}^{c a r, t r}+\beta_{c}^{c a r, l h} \cdot d^{l h}+ \\
& \beta_{c}^{c a r, l m} \cdot d^{l m}+\beta_{c}^{c a r, h d} \cdot\left|\mathcal{S}^{\prime} \cup \mathcal{R}^{\prime}\right|\end{aligned}$ & Costs full transport \\
\hline$C_{t, c}^{c a r, l m}\left(f_{t, v^{c}, c}, d^{l h}, \mathcal{S}^{\prime}\right)$ & $\begin{aligned}= & \alpha_{c}^{c a r, l m}+\beta_{c}^{c a r, l h} \cdot d^{l h}+ \\
& \beta_{c}^{h d} \cdot\left|\mathcal{S}^{\prime}\right|+P_{t}^{u c c, t r}\left(f_{t, v^{c}, c}\right)\end{aligned}$ & Costs outsourcing UCC \\
\hline \multicolumn{3}{|l|}{$\mathbf{U C C}(u c c)$} \\
\hline$P_{t}^{u c c, s b}\left(f_{t, v u c c}\right)$ & $=\alpha^{u c c, s b}+\beta^{u c c, s b} \cdot f_{t, v} u c c$ & Income subsidies \\
\hline$P_{t}^{u c c, r e c, t r}\left(f_{t, v^{r}, r, \gamma \mid \gamma=1}\right)$ & $=\beta^{u c c, r e c, t r} \cdot f_{t, v^{r}, r, \gamma \mid \gamma=1}$ & $\begin{array}{l}\text { Price distribution } \\
\text { (if } r \text { selects UCC) }\end{array}$ \\
\hline$P_{t}^{u c c, c a r, t r}\left(f_{t, v^{c}, c, \gamma \mid \gamma=1}\right)$ & $=\beta^{u c c, c a r, t r} \cdot f_{t, v^{c}, c, \gamma \mid \gamma=1}$ & $\begin{array}{l}\text { Price distribution } \\
\text { (if } c \text { selects UCC) }\end{array}$ \\
\hline$C_{t}^{u c c, r c}\left(f_{t, v u c c}\right)$ & $=\alpha^{u c c, r c}+\beta^{u c c, r c} \cdot f_{t, v}^{u c c}$ & Costs receiving \\
\hline$C_{t}^{u c c, t r}\left(f_{t, v}^{u c c}, d^{l m}, \mathcal{R}^{\prime}\right)$ & $\begin{aligned}= & \beta^{u c c, l m} \cdot f_{t, v}^{u c c} \cdot d^{l m}+ \\
& \beta^{u c c, h d} \cdot\left|\mathcal{R}^{\prime}\right|\end{aligned}$ & Costs distribution \\
\hline
\end{tabular}

To influence their profit, they select a subset of orders to dispatch $x_{t, v}^{u c c}$, and a corresponding route set $\Delta_{t}^{u c c}$ :

$$
\begin{array}{r}
\max \sum_{t \in \mathcal{T}}\left(P_{t}^{u c c, s b}\left(f_{t, v^{u c c}}\right)+P_{t}^{u c c, r e c, t r}\left(f_{t, v^{r}, r, \gamma \mid \gamma=1}\right)+P_{t}^{u c c, c a r, t r}\left(f_{t, v^{c}, c, \gamma \mid \gamma=1}\right)-\right. \\
\left.C_{t}^{u c c, r c}\left(f_{t, v^{u c c}}\right)-C_{t}^{u c c, t r}\left(f_{t, v^{u c c}}, d^{l m}, \mathcal{R}^{\prime}\right)\right) .
\end{array}
$$

Carriers attempt to maximize profit (determined by the transport price, subsidy income, transport costs, and outsourcing costs) by selecting the route set $\Delta_{t, c}^{c a r}$ that minimizes costs at every decision moment. In addition, carriers can choose whether they perform the full transport themselves, or they can decide to outsource last-mile transport to the UCC. For the latter decision, the carriers compare the costs of outsourcing (minus the subsidy income) to performing the last-mile distribution itself, selecting the cheapest solution. Their objective 
function is given by

$$
\begin{array}{r}
\max \sum_{t \in \mathcal{T}}\left(P_{t, c}^{c a r, t r}\left(f_{t, v^{c}, c}, d^{l h}\right)-\min \left(C_{t, c}^{c a r, t r}\left(d^{l h}, d^{l m}, \mathcal{S}^{\prime}, \mathcal{R}^{\prime}\right),\right.\right. \\
\left.\left.\left(C_{t, c}^{c a r, l m}\left(f_{t, v^{c}, c}, d^{l h}, \mathcal{S}^{\prime}\right)-P_{t, c}^{c a r, s b}\left(f_{t, v^{c}, c, \gamma \mid \gamma=1}\right)\right)\right)\right) .
\end{array}
$$

For the receivers and the administrator, we do not explicitly define an objective function, as these agents do not make operational decisions in our framework. The performance of the receiver is measured as the sum of receiving costs. These depend on the tactical decision whether delivery takes place via the UCC. If the receiver does not mandate delivery via the $\mathrm{UCC}$, its costs are given by

$$
\sum_{t \in \mathcal{T}} \sum_{c \in \mathcal{C} \cup \mathcal{H}} C_{t, r}^{r e c, r c}\left(f_{t, v^{r}, r, c}\right)
$$

If the receiver mandates deliveries via the $\mathrm{UCC}$, the receiver pays the $\mathrm{UCC}$ for last-mile delivery, but incur lower receiving costs due to receiving bundled orders from only one carrier. The costs for the receiver are then given by:

$$
\sum_{t \in \mathcal{T}} C_{t, r}^{r e c, r c}\left(f_{t, v^{r}, r}\right)+C_{t, r}^{r e c, l m}\left(f_{t, v^{r}, r}\right)-P_{t, r}^{r e c, s b}\left(f_{t, v^{r}, r}\right) .
$$

The performance of the administrator is measured with the following KPIs: (i) the number of vehicles per type that enter the urban area, (ii) the total distance covered within the urban area per vehicle type, (iii) the income from policies minus the provided subsidies, and (iv) the emission levels for $\mathrm{CO}_{2}, \mathrm{SO}_{2}, \mathrm{NO}_{x}$, and particulate matter $(P M)$. The first two KPIs indirectly capture effects such as noise hindrance and the contribution to road congestion. The third KPI should be a nonnegative number for a financially sustainable scheme.

\subsection{Decision-Making Policies}

All agents aim to optimize their own objective functions. In our dynamic environment, the corresponding operational decision problems are subject to incomplete information. Exact solution methods for stochastic models usually require an unfeasibly large computational effort, which is why we typically resort to heuristic solutions in agent-based simulation. Various policies can be used to tackle the operational decision problems. The specific policies to be used in the simulation are selected based on the instance, and are chosen as simulation settings. For an overview of the possible decisions per agent type, we refer to Fig. 2.

Both shippers and the UCC are faced with the decision when to dispatch accumulated orders. Minkoff [14] describes this problem class as the Delivery Dispatching Problem (DDP). Typical solutions for the DDP are fixed policies based on (i) a threshold on accumulated volume and (ii) the elapsed service time. In our simulation, the dispatching decision is more complicated, as orders are subject to distinct delivery windows, and subsets of orders are periodically 
dispatched. Orders may be held in inventory for a limited time, anticipating future order arrivals for better consolidation opportunities. Dispatch decisions can be significantly improved when taking into account expected future costs [23]. For this DDP variant, methods such as scenario sampling or stochastic modeling are suitable methods to estimate future costs.

Carriers, as well as the UCC, are required to solve a vehicle routing problem (VRP). An abundant amount of studies has been performed on many variants of the VRP. We refer to Cattaruzza et al. [9] and Kim et al. [12] for recent overviews of VRP solution methods in urban logistics; these methods pay particular attention to aspects such as regulations, emissions, and delivery windows. As we split the decision problems into a periodic dispatch decision and a vehicle routing problem, solution methods for the static VRP can be applied. To establish credibility of the simulation model with the stakeholders involved, it is sensible to incorporate algorithms similar to the ones used in practice.

\subsection{Scenario Analysis}

To embed tactical and strategic decisions - requiring commitment on the longer term - into our framework we make use of scenario analysis: measures are given as input to the simulation. In our discussion of scenario analysis, we again refer to the classification of Quak [16]. Governmental policies are typically described by forms of road pricing, parking fees, zone access, and time access restrictions. Such policies are incorporated in the framework by (i) setting constraints as network input, (ii) defining conditional costs and conditions for transport via the urban network, and (iii) specifying allocation rules for possible redistribution in the form of subsidies. Next, we discuss company-driven initiatives. To evaluate measures such as adjusting the fleet or selecting the UCC as the delivery address by the receiver, the characteristics of the agent can be adjusted. Cooperation initiatives are incorporated into the framework as follows. First, the members of the coalition should be specified. Second, the action space of the coalition needs to be defined, including rules for the allocation of tasks to agents. Third, a single objective function must be specified for the coalition. Stable solutions require that the coalitional profit is at least equal to the sum of individual profits of the coalition members. Fourth - as coalitions require rational agents that are willing to cooperate - an appropriate gain-sharing mechanism should be incorporated. Such mechanisms can drawn from the field of cooperative game theory; a comprehensible overview is provided by Osborne \& Rubinstein [15].

Finally, physical infrastructure- and transport-reorganizing initiatives are incorporated by modifying the network and may entail, e.g., positioning the UCC or including special transport lanes for licensed vehicles. It is important that the applied routing algorithms properly take into account such restrictions.

The reliability of the simulation results depends on the scenario input data, which may be subject to high variability. For a complete simulation study, we would propose to first establish a reliable range for each parameter, and subsequently apply a fractional factorial design. Such a design only uses the values corresponding to the range bounds, thereby (i) focusing on the main (interaction) 
effects of measures, and (ii) providing high-level sensitivity analysis. Afterward, more detailed analysis can focus on promising schemes.

\section{Computational Study}

To briefly illustrate the working of our framework, we test a few urban logistics schemes. We implemented the framework as a discrete-event simulation model in Delphi XE6. We represent the city by a virtual 10x10km grid, with 1 UCC located at the edge of the grid, 3 carriers, 10 shippers, and 20 receivers (agents of the same type are identical). Network properties are chosen to sufficiently reflect flexibility and diversity. The line-haul distance between shippers and receivers is $100 \mathrm{~km}$. We consider a planning horizon with 500 decision moments, and perform 5 replications per scenario. A warmup period of 10 time units ensures reaching a steady state. At every decision moment, receivers have a demand between 0 and 5 orders, with order sizes ranging between 0.05 and 0.20 of the capacity of a delivery van. The earliest delivery time varies between 0 and 2 time units; the length of the delivery window varies between 1 and 4 time units. Order properties are generated stochastically, and are subject to uniform distributions.

In Table 2, we show the used vehicle properties. Vehicle capacities and average emission values are obtained from Boer et al. [6], using capacities for voluminous goods and 2020 engine standards. The UCC uses large vans ( $>2$ ton), the linehaul carriers deploy medium-sized trucks $(10-20$ ton). For the delivery van, we deduce costs per hour and vehicle speeds from Roca-Riu et al. [17]. We multiply these costs with 1.5 for medium-sized trucks. We set the transport price charged by the carrier to a fixed shipment fee of $€ 35$ and a variable cost of $€ 1.5$ per $\mathrm{km}$. The UCC charges a volume-based price of $€ 100$ per van-load, and incurs receiving costs of $€ 20$ for every incoming truck. For receivers and shippers, we set receiving- and shipping costs at $€ 5$ per vehicle, respectively.

Table 2. Vehicle properties for carriers (truck) and UCC (delivery van)

\begin{tabular}{lcc}
\hline Vehicle type & Large van $>2$ ton Truck $10-20$ ton \\
\hline Capacity (ton) & 1.2 & 8 \\
Speed line-haul (km/hour) & 50 & 50 \\
Speed urban area (km/hour) & 25 & 25 \\
Handling costs (€/receiver) & 7.9 & 7.9 \\
Costs line-haul (€/km) & 0.83 & 1.24 \\
Costs urban $(€ / \mathrm{km})$ & 1.35 & 2.03 \\
$\mathrm{CO}_{2}(\mathrm{~g} / \mathrm{km})$ & 299.5 & 943 \\
$\mathrm{SO}_{2}(\mathrm{mg} / \mathrm{km})$ & 2.3 & 7.2 \\
$\mathrm{NO}_{x}(\mathrm{~g} / \mathrm{km})$ & 0.55 & 3.1 \\
$\mathrm{PM}_{2.5}(\mathrm{mg} / \mathrm{km})$ & 42 & 56 \\
\hline
\end{tabular}

We now describe the used decision methods. Shippers only dispatch the set of accumulated orders when it contains an urgent order, and holds the shipment 
otherwise. To obtain the expected future costs for the UCC, we use a one-step lookahead policy. We sample 5 random order arrivals, for which we compute the expected future costs per action. We select the dispatch action that minimizes the sum of direct costs and lookahead costs. Finally, to solve the routing problems of the carriers and the UCC, we use the cheapest insertion algorithm. Tactical decisions include selection of the UCC by the receiver, forming a carrier coalition, setting parking costs, and subsidizing the UCC.

We test the following measures: (A) carriers may deliver via the UCC (operational decision), (B) receivers mandate delivery via the UCC (scenario input), (C) carriers form a coalition (scenario input), using the Shapley value as a gainsharing mechanism, (D) parking costs (€3 per stop, only for trucks), and (E) volume-based subsidy to the UCC ( $€ 70$ per full van-load). Based on these measures, we compose and test eight urban logistics schemes. With these schemes, we aim to show how both individual and combined measures affect system performance. In Table 3, we show the results of the agent types for all schemes, stating the financial performance and an indicator for the external costs. Agents that are negatively affected by a scheme (compared to Scheme 1) are marked in bold. The results underline the difficulty to find feasible schemes. Individual

Table 3. Performance of all agent types under various urban logistics schemes.

\begin{tabular}{|c|c|c|c|c|c|c|c|c|c|c|c|}
\hline \multirow[b]{2}{*}{ Scheme } & \multicolumn{5}{|c|}{ Measures } & \multicolumn{5}{|c|}{ Net income $\left(\times 10^{3} €\right)$} & \multirow{2}{*}{$\begin{array}{c}\text { External costs } \\
\text { Admin. }\end{array}$} \\
\hline & A & B & $\mathrm{C}$ & $\mathrm{D}$ & $\mathrm{E}$ & Receiver & Shippe & Carrie & UCC & Admin. & \\
\hline 1 & No & No & No & No & No & -0.6 & -12.9 & 32.9 & 0.0 & 0.0 & $\mathrm{O}$ \\
\hline 2 & Yes & No & No & No & No & -0.4 & -12.9 & 40.0 & -24.9 & 0.0 & ++ \\
\hline 3 & No & Yes & No & No & No & -2.1 & -12.9 & 50.8 & -24.2 & 0.0 & ++ \\
\hline 4 & No & No & Yes & No & No & -0.2 & -12.9 & 67.1 & 0.0 & 0.0 & + \\
\hline 5 & Yes & No & No & Yes & Yes & -0.4 & -12.9 & 40.2 & 5.0 & -28.2 & ++ \\
\hline 6 & Yes & No & Yes & No & No & -0.3 & -12.9 & 68.5 & -11.5 & 0.0 & + \\
\hline 7 & Yes & No & Yes & Yes & No & -0.4 & -12.9 & 67.9 & -12.0 & 1.3 & + \\
\hline 8 & Yes & No & Yes & Yes & Yes & -0.4 & -12.9 & 68.0 & 0.3 & 3.0 & + \\
\hline
\end{tabular}

measures often fail to generate the required commitment from all agents. The funding of the UCC is particularly complex. The correct balance between subsidies and policy income must be found; if carriers mostly deliver via the UCC, the income stemming from parking costs may be insufficient to support the UCC. For the tested instance, the existence of the carrier coalition is required to obtain sufficient income from parking costs. We highlight the results of Scheme 8, which significantly cuts emissions $\left(\mathrm{CO}_{2}\right.$ by $47.5 \%, \mathrm{SO}_{2}$ by $47.4 \%, \mathrm{NO}_{x}$ by $53.1 \%$, and $P M$ by $30.2 \%$ ), reduces the number of trucks in the city center by $60 \%$, and reduce the overall urban transport distance (by both trucks and vans) by $20 \%$. Although many measures have the potential to reduce external costs, the challenge remains to combine them into a feasible scheme. 


\section{Conclusion}

Although the need for improving urban freight transport is widely recognized, existing initiatives often fail due to a lack of commitment by the actors involved. In this study, we designed an agent-based simulation framework to evaluate a wide array of urban logistics schemes. We defined the roles of five agent types, and described their actions, monetary streams, and information streams. For every agent type, we specified KPIs to measure the system performance. To reflect the practice of urban logistics - where we must align the interests of multiple actors - every agent is an autonomous decision maker. As such, we assess the behavior and performance of every agent type. Agents rationally optimize their operational decisions during the simulation, whereas tactical and strategical decisions are embedded by means of scenario analysis.

As practice indicates that schemes combining both governmental policies and company-driven initiatives yield the best results, we designed the framework such that both aspects are well represented. The urban consolidation center (UCC) has a key role in our framework, as it facilitates both consolidation and the deployment of designated delivery vehicles against lower (external) costs. Furthermore, we included governmental policies such as road pricing, zone access and parking costs; gains stemming from these measures can be used to subsidize agents in a closed-loop scheme. To define company-driven initiatives, we described collaboration between carriers, as well as various kinds of interaction between the companies and the UCC. Another distinctive feature of our framework is that we explicitly took into account the effect of line-haul transport on the last-mile distribution, rather than focusing only on the last mile. With our simulation framework, we can measure the impact of schemes on financial performance and external costs, and verify whether autonomous actors could commit to such a scheme in the long run.

\section{References}

1. Allen, J., Browne, M., Woodburn, A., Leonardi, J.: The role of urban consolidation centres in sustainable freight transport. Transport Reviews 32(4), 473-490 (2012)

2. Anand, N., Quak, H., van Duin, R., Tavasszy, L.: City logistics modeling efforts: Trends and gaps - a review. Procedia-Social and Behavioral Sciences 39, 101-115 (2012)

3. Anderson, S., Allen, J., Browne, M.: Urban logistics - how can it meet policy makers sustainability objectives? Journal of Transport Geography 13(1), 71-81 (2005)

4. Bektaş, T., Crainic, T.G., Van Woensel, T.: From managing urban freight to smart city logistics networks. CIRRELT 2015-17 (2015)

5. Benjelloun, A., Crainic, T.G.: Trends, challenges, and perspectives in city logistics. Transportation and land use interaction, Proceedings TRANSLU 8, 269-284 (2008)

6. Boer, E., Otten, M.B.J., Essen, H.: Comparison of various transport modes on a EU scale with the STREAM database. CE Delft (2011)

7. Boerkamps, J., van Binsbergen, A.: Goodtrip - a new approach for modelling and evaluating urban goods distribution. In: Taniguchi, E., R.G., T. (eds.) City Logistics I, pp. 175-186. ARRB Group Limited, Melbourne (1999) 
8. Browne, M., Sweet, M., Woodburn, A., Allen, J.: Urban freight consolidation centres. Transport Studies Group 10 (2005)

9. Cattaruzza, D., Absi, N., Feillet, D., González-Feliu, J.: Vehicle routing problems for city logistics. EURO Journal on Transportation and Logistics pp. 1-29 (2015)

10. Crainic, T.G., Ricciardi, N., Storchi, G.: Models for evaluating and planning city logistics systems. Transportation Science 43(4), 432-454 (2009)

11. Gevaers, R., Van de Voorde, E., Vanelslander, T.: Characteristics and typology of last-mile logistics from an innovation perspective in an urban context. In: Macharis, C., Melo, S. (eds.) City distribution and urban freight transport: Multiple perspectives, pp. 56-71. Edward Elger, Cheltenham, UK (2011)

12. Kim, G., Ong, Y.S., Heng, C.K., Tan, P.S., Zhang, N.A.: City vehicle routing problem (city VRP): A review. Intelligent Transportation Systems, IEEE Transactions 16(4), 1654-1666 (2015)

13. Macharis, C., Milan, L., Verlinde, S.: A stakeholder-based multicriteria evaluation framework for city distribution. Research in Transportation Business \& Management 11, 75-84 (2014)

14. Minkoff, A.S.: A Markov decision model and decomposition heuristic for dynamic vehicle dispatching. Operations Research 41(1), 77-90 (1993)

15. Osborne, M.J., Rubinstein, A.: A course in game theory. MIT Press (1994)

16. Quak, H.: Urban freight transport: the challenge of sustainability. In: Macharis, C., Melo, S. (eds.) City distribution and urban freight transport: Multiple perspectives, pp. 37-56. Edward Elger, Cheltenham, UK (2011)

17. Roca-Riu, M., Estrada, M., Fernández, E.: An evaluation of urban consolidation centers through continuous analysis with non-equal market share companies. Transportation Research Procedia 12, 370-382 (2016)

18. Russo, F., Comi, A.: A classification of city logistics measures and connected impacts. Procedia-Social and Behavioral Sciences 2(3), 6355-6365 (2010)

19. Tamagawa, D., Taniguchi, E., Yamada, T.: Evaluating city logistics measures using a multi-agent model. Procedia-Social and Behavioral Sciences 2(3), 6002-6012 (2010)

20. Taniguchi, E., Thompson, R.G., Yamada, T.: Concepts and visions for urban transport and logistics relating to human security. In: Taniguchi, E., Fwa, T.F., Thompson, R.G. (eds.) Urban transportation and logistics: Health, safety, and security concerns, pp. 1-30. CRC Press, Boca Raton, USA (2014)

21. United Nations: Worlds population increasingly urban with more than half living in urban areas. https://www.un.org/development/desa/en/news/population/ world-urbanization-prospects.html (2014), accessed: 2016-03-22

22. Van Duin, R.J., van Kolck, A., Anand, N., Taniguchi, E.: Towards an agent-based modelling approach for the evaluation of dynamic usage of urban distribution centres. Procedia-Social and Behavioral Sciences 39, 333-348 (2012)

23. Van Heeswijk, W.J.A., Mes, M.R., Schutten, M.J.: An approximate dynamic programming approach to urban freight distribution with batch arrivals. Lecture Notes in Computer Science 9335, 61-75 (2015)

24. Van Rooijen, T., Quak, H.: Local impacts of a new urban consolidation centre-the case of Binnenstadservice.nl. Procedia-Social and Behavioral Sciences 2(3), 59675979 (2010)

25. Wangapisit, O., Taniguchi, E., Teo, J.S., Qureshi, A.G.: Multi-agent systems modelling for evaluating joint delivery systems. Procedia-Social and Behavioral Sciences $125,472-483(2014)$ 